due to gross mechanical canses is often shown by subsequent proceedings that lead to the divorce court, which proceedings may be explained, if not justified, from the biological point of view.

Have we not been unduly cautious and prejudiced in general practice against the use of tuberculin? Its use bas certainly not been generally adopted in this country, although we quickly grasped the utility of using antitoxin in diphtheria. It has been stated that gouty people are never tuberculous. It would be interesting to test the truth of this statement by means of the opsonin test. As medical men all our practice, theory and treatment, is based upon the view that we have to deal with machines out of order; this function has to be put right or that part lopped off. Transcendental medicine or surgery has not yet been written. If vital phenomena are supernatural then our remedies should be supernatural. And where are we to find them? Treatment by suggestion, endeavouring to make patients believe and act upon a delusion, is perilously like playing at insanity.

The life of the individual is the sum total of the activities of his constituent cells. A man is as old as his cells. The cell is the element of living substance. All living substance exists in cells and all the functions of living substance originate in the elementary vital phenomena of the cells. Here we are on sure ground. These are the first principles of medicine. As a working hypthesis the mechanical view of life is the only practical one for medical men. The question of the unity of nature is not called in question.

Hear what a practical bio-chemist says bearing on the point speaking of metabolism. Professor Leathes says : "It implies and gently insists on the idea that all the phenomena of life are at bottom chemical reactions, when a muscle twitches no less than when a gland secretes. It is not too much to say that when we are moved to tears or laughter it is chemical reactions that are the underlying causes to which ultimate analysis must lead us." Who can doubt it who has seen the effects of drugs or other chemical bodies on the moral and intellectual faculties or other manifestations of the nervous system. In myxolema, cretinism, alcoholism, for instance, is it not fantastical to imagine that a material drug can effect a spiritual essence? Poets make much of the paragon perfection of man and argue therefrom his supernatural attributes, but we know from our daily work that man at the present stage of evolution is frequently fearfully and wonderfully badly made-that many specimens are never out of the repair shop. It is probable that in this society there is not a member who has a perfect pair of eyes or whose nasal septum is not out of the perpendicular. In the highest and most important function for the perpetuation of the race (that of reproduction) the complaint that is continually poured into our ears is that of inefficiency.

The question of diet bulks largely in any scheme of treat ment. Perhaps the burning question of the hour is what amount of proteid is necessary and advisable to maintain a high standard of health? The question cannot be answered with any certainty at present but we know by direct experiment under different conditions, as well as by the habits of some hardy and intellectual races, that great physical and intellectual activity is compatible with much less than the conventional dietary of 100 grammes or more of proteid, and that particularly in persons whose ancestors have for generations taxed to exbaustion point the cells concerned in nitrogenous metabolism a great part of this proteid may be advantageously replaced by carbohydrates or other nonnitrogenous foodstuffs, the more especially because much of the proteid never reaches the tissues as proteid. He is a wise man who knows his own metabolic limitations and does not attempt to emulate men with primitive digestions. With new drugs and new remedies we are apt to forget the debt we owe our medical forefathers who by sheer therapeutic intuition discovered and handed down to us such valuable remedies as opium, cinchona bark, mercury, nux vomica, vaccine, and coca leaves, which the Mexicans used for generations as a local anæsthetic before the alkaloid was discovered. Coal tar was the forerunner of, and used for the same purposes as, carbolic acid. Carbolic acid gave birth to salicylic acid. The pine-forest cure suggested eucalyptus oil. I have to confess to a sneaking affection for the oldfashioned, much-ridiculed blunderbuss prescription. Eich tissue knows its own requirements. It may be that I was prejudiced in the favour of these prescriptions in my early apprenticeship days. But, after all, complexity is a higher stage of evolution than simplicity. And nature's remedies are all complex. Take, for instance, the natural mineral waters, or opium or cinchona. They are all blunderbuss prescriptions. Even the simplest fare, such as bread-andmilk, contains over 20 ingredients and yet each individual ingredient is unerringly conducted to its affinitive tissue.

We may hope that as pharmacology advances that mechanical or empirical prescribing will become less and less frequent. At present we know that drugs produce their effects either by chemical combination or by their physical properties interfering with oxidation processes, as sand puts out a fire, and their ability to penetrate a cell may depend upon the size of the molecules of which they are composed. Instances of chemical combination are shown in the exhibition of alkalies in diabetic coma and sulphonal poisoning, in the action of sulphate of soda in carbolic acid poisoning, and of sodium hyposulphite in combining with prussic acid to form the non-poisonous sulphocyanide. The most powerful narcotic substances are those which combine a very slight solubility in water with a very high solubility in ether, olive oil, or brain lipoid. The cerebral cells are more sensitive to alterations in their composition than other cells and they contain a much larger percentage of "lipoid matter" or fat, so that these drugs tend to accumulate in them. Here we have an instance of the physical action of remedies. To this class belong the trional group. As a practical point 1 may remind you that the effects produced by adrenalin upon any tissue are such as follows excitation of the sympathetic nerve which supplies the tissue. Consequently, in cases of hæmorrhage from the lungs it is contra-indicated, because the muscles of the pulmonary blood-vessels are not supplied with constrictor fibres.

And now, Gentlemen, in conclusion, I fear that the limits of your patience must be reached. I am only too conscious of having rushed you from China to Peru, and only hope that on the voyage you may have annexed here and there some trifling bric-a-brac to furnish your study. The late Dr. Abercrombie sums up the lesson that I would take to myself and offer to you this evening. He says: "In regard to the effect of medicines in particular, there are two opposite errors to be equally avoided-namely, an implicit confidence in the power of pariicular remedies in every case, and a total scepticism in regard to the resourses of medicine. Both these extremes are equally unworthy of persons of calm philosophical observation, and they who advance carefully in the middle course, not misled by the temptation to basty conclusion, and cautioned but not discouraged, by the danger of concluding falsely, are most likely to contribute something towards diminishing the uncertainty of medicine."

Authorities cited.-Prollems in Animal Metabolism (Leathes). Recent Advances in Bio-Chemistry and Physiology (Leonard Hill), General Physiology (Verworn), Pharmacology (Dixon), and Essays (Chittenden)

\section{ON OPSONINS AND TREATMENT BY BACTERIAL VACCINES.}

By J. I. BUNOH, M.D., D.SC. LOND, M.R O.P. LoND., IATE RESEARCH SCHOLAR OF THE BRITISH MEDICAL ASSOCIATION.

THE advances of science, like those of nature, are slow, and the marvellous discoveries which are introduced to the public in their completed form and then appear, like Pallas Athene, to have leapt fully developed from the head of their creator, can on closer examination be traced through all the stages of their gradual development. Although, to the romantic imagination, the falling of an apple, the swinging of a church lamp, and the flowing of water from a bath revealed to Newton, to Galileo, and to Archimedes respectively the secrets of nature, nevertheless, truth compels us to recognise that these secrets were only unravelled by ceaseless striving and strenuous work, and that only by similar means can future advances in the field of research be accomplished. Thus we can trace back to far distant times the first attempts at preventive inoculation. It was early recognised that certain diseases occur only once in the life of the individual and he is in future protected against attacks of the same disease. From this it was a short step to the artificial production of such diseases and it has been stated that the practice of artificially inoculating small-pox

1 A paper read before the Medical Society of University College, London, on Nov. 14th, 1906. 
was known and practised thousands of years ago by the Indians and Chinese, and was quite a common practice in the Middle Ages. As time went on there was evolved the method of inoculation of cow-pox to protect against smallpox and, more recently, the use of antitoxic sera and various forms of vaccines to neutralise the effects of diseases which had already invaded the organism. The cure by such means of an already established disease is, of course, of the greatest importance to the community and various explanations have been given of the destruction of bacteria within the organism.

According to some, it depends on some action of the serum, according to others on the activity of the phagocytes which ingest and destroy the bacteria, while others hold that both serum and phagocytes are necessary. Behring showed that anthrax bacilli grow well on the blood serum of animals which are susceptible to anthrax inoculation but cannot be got to grow on the serum of white rats which have been rendered immune to anthrax. He also showed that while one particular serum is fatal to certain varieties of microorganisms it is powerless to inhibit the growth of certain other organisms and that a definite amount of serum is only capable of dealing with a certain definite number of bacteria, so that if bacteria in excess of this number are introduced they grow and multiply actively. In other words, the immunity thus conferred depends upon the ratio between the number of micro-organisms and the quantity of the substance inimical to their growth contained in the fluid.

It has been shown that the destruction of the typhoid and cholera bacillus can be brought about by lysins which are present in the corresponding immune sera after artificial inoculation (Pfeiffer) and lysins have been assumed to exist in other diseases also. In the case of diphtheria and tetanus antitoxins are employed clinically which neutralise the respective toxins. Anti-bacterial substances have by some been held to exist within the phagocytes in the form of ferments and in certain circumstances to be secreted by them. According to Metschnikoff the destruction of bacteria is carried out by the phagocytes alone which have in the case of natural immunity been pictured as carrying on a war with the microbes that are attempting to invade the organism. Relying on the experiments of Bordet and Gengou be ascribed the whole protective influence to the phagocytes alone, but it has since been conclusively shown that these, washed absolutely free from serum and the substances contained in it, have of themselves no phagocytic action on bacteria.

The theory, which is a combination of Metschnikoff's phagocyte theory and Behring's humoral theory, receives strong confirmation from some experiments of Buchner. By suitable chemical stimulation he was able to cause the secretion into the pleural cavity of rabbits and dogs of leucocyte-containing exudations which possessed bactericidal powers stronger even than those of the blood or serum of the same animal. Under these conditions, however, phagocytosis could be observed to be proceeding actively and the question immediately arose as to how far the bactericidal qualities of the fluid depended upon phagocytosis and how far upon the contained alexins or anti-bodies. The question seemed to be settled by freezing the fluid and by this means killing the leucocytes but not injuring the alexins, and then again testing its bactericidal powers. These were found to be in no way diminished, and, inasmuch as the leucocytes had been killed, the capacity of the fluid to destroy bacteria must necessarily depend upon the contained anti-bodies. But even if phagocytosis is merely a secondary phenomenon and the destruction of bacteria is brought about by poisonous substances circulating in the fluids of the body, there is a considerable amount of evidence which goes to show that these poisons are secreted by the leucocytes. The influx, therefore, of these white corpuscles to an infected area means an increased secretion of substances poisonous to the invading micro-organism and increased powers of resisting disease. One substance, opsonin or immune body, is, together with the complement. present in every normal serum and accounts for its bacteriolytic and hæmolytic action. Numberless observers have shown the presence of such a substance, which becomes attached to the bacteria in such a way as to render them capable of absorption and destruction by the phagocytes, but whether this action is entirely due to immune body or whether free complement also takes part in the preparation of the bacteria is not so certain. It would, at any rate, increase the strength of action of the immune body, even if it were not absolutely necessary. I have drawn no distinction between opsonin and immune body, not because they are identical, but because up to the present it has not been shown that they are different. It has been asserted by Wright and Douglas that opsonins are thermo-labile-i.e., destroyed by heat-and therefore differ essentially from immune body. They state that if the serum be heated to $60^{\circ} \mathrm{C}$. for a quarter of an hour and a tube containing the usual proportions of washed corpuscles, bacterial emulsion, and heated serum be incubated for 15 minutes no bacteria will have been ingested by the phagocytes becanse the opsonin necessary for their preparation has been destroyed by heat. If this were true it would make it probable that the opsonin is either of the nature of complement or receptor of a fourth order. Dean has, however, in an admirable piece of work, shown that when fresh serum, either normal or immune, is heated to $60^{\circ} \mathrm{C}$. for various periods there is a great fall, as estimated by Wright and Douglas's method, in the amount of activating substance in the first two minutes and after that the curves run almost parallel to the base line. In the case of normal serum the fall is so great that the curve may reach the base line. The fall in the curve does not, however, indicate complete destruction of the substance, as was stated by Bulloch and Atkin, but merely indicates that the substance has reached a concentration below that demonstrable by Wright and Douglas's method. Horse serum after heating to $60^{\circ} \mathrm{C}$. for as much as four hours still contains a considerable amount of the substance, so that, as judged by the effect of heat, there is no reason to distinguish between the opsonin of normal serum and the active substance of immune serum.

Whatever the exact nature of opsonins, they undoubtedly act on the bacteria in such a way as to enable them to be taken up by the phagocytes. This is proved by the following experiment of Wright and Douglas. Two tubes are incubated for a quarter of an hour at $37^{\circ} \mathrm{C}$, the ore containing three volumes of normal serum, plus one volume of staphylococcus emulsion (previously heated to $75^{\circ} \mathrm{C}$. and cooled), the other containing three volumes of serum which has been heated to $60^{\circ} \mathrm{C}$., plus one volume of staphylococcus emulsion (previously heated to $75^{\circ} \mathrm{C}$. and cooled). On removal from the incubator the first tube (containing normal serum) is heated to $60^{\circ} \mathrm{C}$. and cooled. The contents of each tube are then added to three volumes of white blood corpuscles and the number of staphylococci taken up per phagocyte counted. It is found that the number of staphylococci taken up in the second case is only one-seventh as compared to that taken up in the first case; in other words, the opsonin acts directly on the bacterium. It seems that opsonins are specific and in a considerable number of infections specific opsonins have been demonstrated. Variations in the amount of opsonins in the blcod are not necessarily associated with variations in the number of leucocytes.

An increase of the opsonic content of the blood serum can be brought about by the injection into the organism of vaccines. Of these the best known is the "old" tuberculin of Koch, which he employed as an injection in the treatment of tuberculous patients when first he introduced the tuberculin method some 15 years ago. The preparation is a clear brownish fluid, somewhat viscid from the presence of glycerine, and is diluted down before use according to the required dose, bearing in mind that the dilutions must be freshly prepared. It is obtained by growing the tubercle bacilli in glycerine-veal broth in the presence of a sufficiency of oxygen for from 6 to 12 weeks, and then sterilising, evaporating, and passing twice through a Chamberland filter. The resulting fluid is practically a solution in glycerine of the cellular metabolic products of the organism and contains one or more toxic bodies which may be either of the nature of a ferment, albumose, or alkaloid. It is used chiefly for diagnostic purposes in doses of one cubic millimetre, which in tuberculous patients produce a rise of temperature and inflammatory reaction of neighbouring tuberculous lesions. If this reaction is absent double the dose is given two days afterwards and, if there is still no reaction, double the last dose is given after another two days. If reaction does not show itself after the third dose the patient is probably not tuberculous. Although this "old" tuberculin is sterilised and there is therefore no danger of inoculating the patient with tubercle by its use, it is much less used for therapeutic treatment than tuberculin Koch T.R, which is prepared from a virulent culture of tubercle by drying, grinding up the 
oacilli, and, by washing, removing the extracellular toxins which chiefly constitute the " old" tuberculin. The residue is then made into an emulsion with distilled water after trituration, centrifugalised, and the supernatant fluid pipetted off. To it 20 per cent. of glycerine is added to preserve it and it is standardised so that one cubic centimetre contains ten milligrammes of solid substance. Dilutions can then be made to any required dose and these are heated for an hour at $60^{\circ} \mathrm{C}$. in capsules so as to kill any possibly contained living bacilli. The dose is to commence with seldom more than $\frac{1}{10} 0^{\text {th }}$ milligramme, usually less, and is injected subcutaneously with strict aseptic precautions. The injection should cause no rise of temperature and no general or appreciable local reaction. The amount of the dose given at subsequent injections and the intervals between the doses depend on the influence produced by the preceding dose on the opsonic index to tubercle, but the doses of one-half, one, or two milligrammes which were tormerly given, are now never employed. Tuberculins have also been prepared by other pathologists, by modifications of the methods given above, or by emploving hydraulic pressure to cultures of tubercle, or by oxidising them with hydrogen peroxide so as to convert the toxins into antitoxins. But the tuberculins of Hahn, of Denys, of Béraneck, of Buchner (known as tuberculoplasmin), of Landmann (known as tuberculol), of Hirschfelder (known as oxytuberculin), and of Klebs (known as tuberculocidin and anti-phthisin) are seldom used in this country.

The elaboration of protective substances in the organism can be induced by chemical substances or vaccines prepared from bacteria other than the tubercle bacillus. I myself have used and injected vaccines of pneumococci, streptococci, and the various forms of staphylococci during the past 18 months. These vaccines are emulsions of that species of organism which has produced the disease in the patient and, inasmuch as different strains of the same organism differ greatly, the vaccine should, when possible, be prepared from a pure culture of that particular organism grown directly from the lesion to which it has given rise in the patient. In some diseases this can be done only with difficulty. In pneumonia, for instance, a mouse must be inoculated from the patient's sputum or empyema pus and a pure culture of the pneumococcus obtained from the mouse's heart blood, and a vaccine made from the culture. The emulsion prepared from such a culture must be centrifugalised and standardised so that a certain volume contains the dead bodies of a certain known number of bacteria, plus their products of metabolism. The initial dose must always be small since patients react very differently to injections and it is essential not to induce too marked or too prolonged a negative phase. This is true of any injection of a vaccine, whether it be the first or a subsequent one, but the reaction of any particular patient to a certain vaccine can be gauged from the alteration of his opsonic index after the first dose.

The inoculation of a vaccine is followed always by a certain definite train of events which, according to Wright, is as follows: 1. Upon the inoculation of a vaccine there supervenes a period of intoxication which is characterised by a decline in the anti-bacterial power of the blood. This "negative phase" is more or less accentuated and prolonged according as a larger or smaller dose of the vaccine is inoculated. In the former case the "negative phase" may disclose itself to clinical observation by a temperature reaction and constitutional disturbance. In the latter case the "negative phase" may be quite unaccompanied by clinical symptoms. 2. Upon the "negative phase" there follows a "positive phase." This phase, the characteristic feature of which is an increase in the anti-bacterial power of the blood, corresponds to a period of increased resistance. A curre which shows graphically the changes in the antibacterial power of the blood runs up in many cases to a sharp peak and sinks away first comparatively rapidly and afterwards more slowly. There are associated in many cases with the climax of the " positive phase" a sense of increased physical vigour and a very pronounced feeling of well-being. 3. After the negative and positive phase the blood may be maintained for a variable period (after tubercle inoculations occasionally for as long as a month) at a somewhat higher level of anti-bacterial power than before inoculation. Orand this in connexion with inoculations of tubercle vaccine is a more usual event-the anti-bacterial power of the blood may over and over again fall back after ten days or a fortnight to the level at which it stood anterior to the inoculation.
If the right dose has been given the negative phase will not be too marked or unduly prolonged and will soon be followed by a well-marked positive phase, and in succeeding inoculations the negative phase will be still less. But if too large a dose be given or it be injected when the index is falling the positive phase will make its appearance very late or not at all. I purposely lay stress on this point because some of the first injections which I gave more than 18 months ago were too large, and I have known an injection of $5^{\frac{1}{\delta}} \mathrm{th}$ or $\overline{4}^{1} \sigma^{\mathrm{t}}$ th milligramme to be followed by a negative phase which was so marked as to last for weeks. It seems to be generally agreed that only the minimal dose must be employed which will produce a satisfactory response and this must not be increased until it ceases to produce its effect. The dose, moreover, must not be repeated until the effect of the preceding dose is passing off. If it were possible to produce a summation of positive phases it would be advantageous to give successive doses at short intervals, so as to produce a maximal favourable effect, but such summation is not possible in the case of tubercle. Each inoculation, therefore, produces its effect independently, and it remains to take the greatest advantage possible of the increased elaboration of protective substances which the artificial stimulus has produced. It is obvious that this can only be done by bringing the protective substance as closely as possible into contact with the bacteria which are the cause of the disease. This is simple enough when the bacteria lie within easy access of the blood stream, but when they are flourishing in the tissues in circumstances most favourable to their growth, remote from the blood stream and in contact only with a lymph which is naturally poor in anti-bacterial substances, the mere increase of the opsonic power of the blood is not sufficient. The opsonin must be brought into direct action with the bacilli in the diseased focus, and this can only be done by increasing the lymph flow through that focus. When it is situated near the surface this may best be brought about by the application of counter-irritants, rubefacients or beat, either dry or moist, in the form of hot-water bottles, hotwater tins, or the commonly used hot boric fcmentations. When the focus or disease is in a joint Bier's method of passive congestion, used with so much success in this hospital by $\mathrm{Mr}$. A. $\mathbf{F}$ J. Barker, is one of the greatest service. The effusion of lymph produced by each of these methods is very considerable, and, in addition, the viscosity and coagulability of the blood can be greatly diminished by the internal administration of decalcifying agents, such as citric acid. The diseased tissues can thus be irrigated by an increased flow of $1 \mathrm{ymph}$, and if this lymph contains an amount of protective substance which has been artificially increased by means of vaccines the fight against the disease germs is carried out under conditions which are much more likely to lead to their destruction. In some cases the surgeon can render the greatest assistance by operative removal of part or the whole of the diseased focus. It is, however, to be borne in mind that in the case of abscesses cure is not brought about merely by the evacuation of the pus and the bacteria contained in it, but rather by opening up the channels which have been closed by the pressure and the vessels which have been blocked, so as to re-establish the stream of blood and lymph containing protective and antibacterial substances. That these are normally wanting in the pus of an abscess can easily be shown by centrifugalising the fluid and proving that the liquid portion contains no opsonin. Apart from artificial increase of protective substance by injections of vaccines we must always remember the possibility of a similar increase by meaus of antoinoculation, but localised bacterial infections give rise to them very scantily or not at all. Such infections run a chronic course over long periods of time, and do not tend to get well. In such cases the protective substance, if it is to be increased at all, must be increased artificially.

The opsonic index having been determined, it remains to deduce from this some conclusions as to the patient's state. If the case be one of suspected tubercle and the opsonic index to tubercle be as much as 30 per cent. above or below normal, a positive diagnosis may be made. An index to tuhercle below 08 is undoubtedly abnormal and indicates either a strong disposition to infection such as is to be expected in a member of a family several of whom have already shown signs of pulmonary tuberculosis, or that infection has already taken place. There is not sufficient evidence to show whether the low index is necessarily the result of infection in any particular case, but this is probable if the index determined 
from time to time shows considerable variations. From the point of view of diagnosis, where tubercle bacilli cannot be found in the sputum, such a series of observatio is showing the presence or absence of periodic variations in the index is of the greatest importance and may be the means of the disease being arrented and the patient cured before the bacterial infection has assumed serious proportions, in other words, we may by this means be able to make an earlier diagnosis than would otherwise be possible and send the patient to a sanatorium in time to effect a rapid cure. Some observers think, however (and I agree with them), that a low index may precede infection, and even be the cause of infection, owing to an inborn or acquired inability to manufacture a sufficient quantity of protective material. From the point of view of prognosis a low index necessitates a very guarded prognosis, either as regards the possibility of infection or the relapse of a so. called sanatorium cure. Bulloch examined 14 picked cases of such cures and found their resistance low in every case except one, and of these cases one, which was considered a perfect cure, relapsed within a year.

An index appreciably above normal means, as a rule, that infection has already taken place, especially if repeated estimations show considerable fluctuations of the opsonic index. Julloch estimated the opsonic index in 44 healthy medical students and 40 healthy hospital nurses, and for the 84 individu $t$ ls he got an average index of 0.96 . The variations in health range from 08 to 1.2 . A phthisical patient, however, may have an index considerably more abnormal than this. It may be more than 50 per cent. below, or more than 100 per cent. above, normal and, in addition, the patient's in tex to staphylococci, streptococci, or pneumococci, or even to all three, may at the same time be quite abnormal. In such a case there is, of course, a mixed infection. In acute recent cases the index to tubercle is geverally low, whether it be a lesion of the lungs, joints, or skin, because the focus of disease has not been shat off by fibrous or granulomatous tissue and toxic material is absorbed at varying intervals into the circulation and negative phase succeeds negative phase or becomes superimposed on preceding negative phase. Where the immune body is deficient, the patient's general health bad, and resisting power low the index is low, and a very small amount of tuberculous toxin is sufficient to produce a marked depression of the opsonic index. If, however, the tuberculous lesion is of old standing and has to a large extent become isolated bp fibrous tissue and shut off, as it were, outside the organism the opsonic index may be considerably above the normal. In such a case the doses of toxin which enters the circulation are small, and after each such auto-inoculation the resulting negative phase has time to be succeeded bp a positive phase. In fact, the patient's protective mechanism is working under fairly satisfactory conditions and if it can only be stimulated to produce sufficient opsonins the tubercle bacillus may be worsted and a cure of the patient effected. But it is essential for success that the autoinoculations shall be properly spaced and shall not succeed one another before the negative phase which succeeds each inoculation has passed off. When the bacterial infection is localised, as in chronic lupus and many cases of boils and acne, little if any auto-inoculation occurs and there is no constitu ional disturbance due to the microbe, so that in such cases most excellent results can be obtained by means of vaccines. In addition to such injections the patient's general health must be improved and the conditions which have rendered him liable to infection removed so far as possible, so as to increase the natural as well as the artificial immunity brought about by the inoculations.

But tubercle of the lungs is in a different category. One has known so many of these cases lose ground, even when treated early and under the most favourable hygienic conditions in Swiss health resorts and elsewhere, because they are inoculating themselves from their pulmonary lesion and from carelessness and inattention to details these auto inoculations are allowed to succeed one another too rapidly, cau-ing superposition of negative on negative phase. The constitutional disturbance which results from such a localised bacterial infertion of the lungs shows itself in pyrexia, weakness, and anæmia, and when the tuberculous infection is accompanied (as is usually the case in phthisis) by streptococcic, staphylococcic, or pneumococcic infection these symptoms are still mure marked. Such a patient should be placed at rest in a pure atmosphere as free as possible from germs and under the best obtainable external conditions until his lung mischief has quieted down and auto-inoculations have so far as possible ceased and his opsonic index become fairly constant. Small doses of tuberculin may then be injected and the effect on his opsonic index estimated, at first frequently and afterwards at such intervals as will enable the duration and degree of the negative and positive phase caused by each injection to be khown, having regard also to the possibility of intercurrent auto-inoculations, each of which would, of course, until its effect had passed off, prohibit the injection of tuberculin. Where it is possible to determine the nature of a mixed infection, vaccines prepared from the organism which is associated with the tubercle bacillus in the patient should be employed.

Tuberculous affections, in which surgical treatment is necessary, can be considerably benefited by simultaneous injections with tuberculin. Through the kindness especially of Mr. R. J. Godlee, of Mr. B. Pollard, and of Mr. R. Johnson, I have been able to watch the effect of such treatment in some of their cases in University College Hospital and I take this opportunity of thanking them most cordially. It must be remembered that the effect of a surgical operation on a tuberculous focus is to throw some of the toxin into the circulation and so depress the resistance of the individual. This negative phase is necessarily disadvantageous to the patient, even if it does not directly interfere with the healing of his wound, or, coinciding with an accidental auto-inoculation of the patient, lead to general tuberculosis. If, however, the opsonic index be raised before operation by suitable injections of tuberculin, the negative phase due to the operation becomes superimposed on a positive phase produced by artificial inoculation and its injurious effects are masked. Tuberculin administered after operation may not only assist in the immediate recovery of the patient but may also help to prevent recurrence of the disease, especially if it has been found impracticable to remove every tuberculous focus. Such injections must, of course, be kept up over a considerable period of time.

I should like to refer briefly to two cases of Mr. Godlee's which were operated on in January, 1906, and have given excellent results. The first was a woman, aged 34 years, living in the country. who had lupus of the left cheek for 26 years. She had been under treatment at a skin hospital for two and a half years and had had about 600 exposures to $x$ rays as well as much other treatment without curing the lupus. When she came under Mr. Godlee's care she had a patch of lupus about three inches long by two inches broad, superficially ulcerated and merging into scar tissue abont an inch wide at the lower edge. There was a small outlying nodule on the left ala nasi. Brown pigmentation and dilated venules were present on the side of the face and lips as a result of the $x$ rays. On Jan. 23 rd her opsonic index was 0.58 . She was given an injection of ${ }_{10} \sigma_{0}$ th milligramme of tuberculin. On Jan. 31st her opsonic index was 076 , and on this date she was operated on by Mr. Godlee. An incision was made all round the affected area of the skin and the included area dissected off the deeper structures. Bleeding was stopped by torsion, hot water, and adrenalin. The raw surface was covered in by Thiersch grafts cut from the front and inner side of the right thigh. On F+b. 1st her opsonic index had fallen to 062 and on the 7 th she was given a second injection of tubtroulin and again on the $13 \mathrm{th}$ and 20 th. On the 27th the skin graft had completely taken and she left the hospital for her home in the country. Since then she has attended at the hospital from time to time and has had four more injections of tuberculin, the last dose of $\frac{1}{2} \frac{1}{5} \sigma$ th milligramme on Sept. 11th, when her opsonic index was $0 \cdot 94$. There was no recurrence of the lupus.

The sr cond case was that of a man, aged 38 years, who had alreadv had one tuberculous testicle removed and was admitted under Mr. Godlee's care to have the second testicle removed and a tuberculous ulcer of the tongne excised. This was done on Jan. 31st, 1906. In the skin of the arms and trunk were the scars of nodules (presumably tuberculous) which had alrearly been cut out. Before operation his opsonic index was 062 and the day after operation it was 051 . On F. b 5th it was 0.58 , and on the $7 \sin$ he had an injection of 1 th milligramme of tuberculin, which was repeated three tımes duing his stay in hospital, his opsonic index on March 7th being 1.1. This gradually dropped to $083 \mathrm{nn}$ July 31st, when he came to London and was given an injection of ${ }_{10}^{1} \sigma^{\text {th }}$ milligramme. He writes that he is feeling well, is at work, and has had no recurrence since leaving the hospital. 
A case of Mr. Pollard's is also of interest in that the tuberculous affection was acute when the patient came under his care.

A girl, aged 11 years, was admitted to University College Hospital on May 18th, 1906, and discharged on June $27 \mathrm{th}$ cured. Three years ago she was seen in the out-patient department by Mr. Pollard for signs of tuberculous disease of the left hip. She was put on a Thomas double hip splint and remained on it for a year. The symptoms gradually subsided and the disease appeared to have cleared up. She was kept lying down for another six months, then she was up and about. Since then, during the last six months, she had several bad falls and the mother attributed the present condition to these falls. When admitted the right hip was greatly swollen and there was a large abscess on the anterior and outer parts of the joint. The skin over the abscess was slightly red. The hip-joint was quite fixed in good position, the limb being slightly abducted and rotated out. There was some tilting down of the pelvis on the right side with one and a half inches apparent lengthening, but in reality half an inch shortening. There was no sign of disease in any other joint or organ. On May 19th Mr. Pollard operated by the usual incision for an anterior excision of the hip. The acetabulum was found to be perforated and an abscess inside the pelvis was evacuated, the diseased bone scraped and a counter opening made at the lower part of the abscess and two tubes inserted. She suffered considerably from shock. On admission the patient's temperature was $100 \cdot 6^{\circ} \mathrm{F}$. but after the operation it did not rise above $100 \cdot 2^{\circ}$. Seven days after the operation she was given

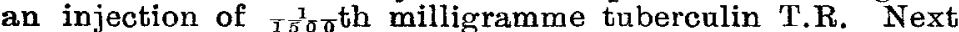
day her highest temperature was $99 \cdot 2^{\circ}$ but after that it did not rise above $99^{\circ}$. Six days after this injection and 13 after the operation, a second injection of ${ }^{1}$ th milligramme tuberculin was given, and again a third dose on June $22 \mathrm{nd}$, her temperature now being normal, her appetite good, and only small granulating surfaces present where the tubes had been. On June 27th she left the hospital and went to the seaside, her opsonic index then being 1:1 as compared with 0.68 when first examined. From there she came to the hospital at the beginning of August and was given a further

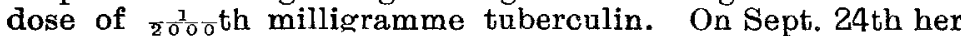
opsonic index was 0.92 and she is apparently quite cured.

My thanks are due to Mr. P. M. Heath, surgical registrar, for the clinical notes of these cases.

It is, of course, possible that these cases might have done as well without tuberculin, but their past history was in each case unfavourable and showed a tendency to recurrence, while their power of resistance to the tubercle bacillus was low. This was increased by tuberculin injections, and it is reasonable to suppose that their rapid recovery was at least aided by increasing their resisting power to the bacterial infection. I will not weary you with any more cases, but I might refer you to a paper in THE LANCET of Oct. 27th, 1906 , p. 1130, by Turtor and Parkin of Hull giving details of 34 cases of various kinds which they have recently treated in their practice, in 30 of which the injection of bacterial vaccines gave excellent results. The results of treatment of some dermatological cases have already appeared in a paper by Pernet and myself in the October, November, and December numbers for $19 \mathrm{C} 6$ of the British Journal of Dermatology. So many other successful cases have now been recorded that the employment of bacterial vaccines must in the future prove of the greatest service in the treatment of disease when controlled by the method which Wright and Douglas have so ably elaborated. They must in many cases be used in conjunction with other methods, for as long as a patient has a focus of disease he is liable to reinfection. In conclusion, I have to express my indebtedness to Dr. W. Bulloch for much valuable advice and assistance and to Professor Sidney H. C. Martin for his kindly encouragement and for permitting me to avail myself of the resources of his laboratory.

Park-street, Grosvenor-square, W.

Literary Intelligence.-Mr. James Stanley Little, from whose pen we publish this week an interesting lay view upon the Ri iera as a health resort, is publishing through Messrs. W. $H$ and L Collingridge a new work entitled "The Doom of Western Civilisation." Mr. Little's title is probably more pessimistic than his book, for his previous writings lead as to believe that he thinks a good deal of vitality still remains in the British Empire.

\section{A CASE OF HæMORRHAGIC CYST OF THE SPLEEN.}

By W. L. HARNeTT, M.A., M.B. Camb., F.R.C S. ENG., ASSISTANT PATHOLOGIST AND DEMONSTRATOR OF MORBID ANATOMY, ST. THOMAS'S HOSPITAL.

THE patient, a male, aged 40 years, was admitted into St. Thomas's Hospital under the care of Mr. Clutton on Nov. 25th, 1905. He was at that time a coal porter, but had been in the Marines for 21 years and had served abroad in various parts of the world for, in all, 15 years. There was a clear history of malaria, but he denied having ever had syphilis. The family history was of no interest. Seven weeks previously to admission he noticed a small swelling on the inside of the left thigh just above the knee. This increased in size so rapidly that in less than a month he was unable to walk. On admission a pulsating swelling, having all the characters of an aneurysm of the popliteal artery, was present on the inner side of the left thigh just above the knee. The leg was markedly wasted and, in common with the rest of the body, was covered with a pustular rash of a scabetic character which, according to the patient's statement, had been present for some time. There was wellmarked general arterio-sclerosis and no pulsation could be felt in the peripheral arteries below the aneurysm. The liver and spleen could be felt to be enlarged. The urine contained a trace of albumin. During the few days preceding operation the aneurysm increased rapidly in size, the circumference of the thigh, which on admission was $14 \frac{1}{2}$ inches, rising to $15 \frac{3}{4}$ inches three days later. At the same time the sk:n over the aneurysm began to show signs of pressure. On Nov. 28th Mr. Clutton operated and ligatured the femoral in Hunter's canal, the walls of the artery being found to be extensively diseased. During the next few days the temperature assumed a septic type, with signs of suppuration in the neighbourhood of the aneurysmal sac, and at the same time suppuration became more active in the pustules on that leg. There were no signs of gangrene in the leg, the state of the circulation being as before the operation. On Dec. 3rd an incision was made over the sac without actually opening the latter and extensive cellulitis was found. As will be detailed below, suppuration had actually taken place in the sac, the infection having presumably reached it from the pustules in the overlying skin, the ligation having probably hastened events by diminishing the klood-supply to the parts. After the incision the temperature fell but death took place two days later.

Post-mortem examination.-Only a partial necropsy was allowed. The skin over the aneurysm was in a sloughing condition and the sac was found to be filled with suppurating blood clot. The femoral and popliteal arteries were in a state of advanced atheroma with extensive calcification. The lumen of the femoral was firmly occluded by the ligature. The heart was enlarged, mainly owing to hypertrophy of the wall of the left ventricle. Both mitral and aortic valves were thickened, but were competent when tested in the usual manner; there were no recent vegetations upon any of the valves. There was well-marked atheroma of the aorta, though not so extensive as might have been expected from the condition of the femoral and popliteal arteries. The peritoneum and intestines were normal. The liver was enlarged, weighing $86 \frac{1}{2}$ ounces, and was pale in colour and markedly cirrhotic. In addition, there were several deep puckered scars, resembling those caused by the healing of gummata. The cut surface showed the fibrous tissue to be increased, dividing up the parenchyma into islets in the manner usual in polylobular cirrhosis. There was slight enlargement of the kidneys, the organs weighing $7 \frac{1}{4}$ and 7 ounces respectively. The capsules were adherent and on stripping them a coarsely granular surface of an unusual appearance was left, the elevated areas being of a dead white colour, in striking contrast to the darker coloured depressed portions. The amount of cortical substance was relatively diminished and was of a white colour, whilst the pyramids were dark. The renal arterioles were moderately thickened. The spleen was converted into an enormous unilocular cyst, measuring about 10 inches by 6 inches, of a dark indigo colour, with an irregnlar bossy surface. There were numerous adhesions, of considerable standing, between the peritoneal surface and the surrounding organs. On section the cyst was found to contain nearly a pint of dark, brownish-red, turbid fluid, 